\title{
Diverse small molecules prevent macrophage lysis during pyroptosis
}

\author{
Wendy P. Loomis', Andreas B. den Hartigh', Brad T. Cookson ${ }^{1,2}$ and Susan L. Fink (i)
}

\begin{abstract}
Pyroptosis is a programmed process of proinflammatory cell death mediated by caspase-1-related proteases that cleave the pore-forming protein, gasdermin D, causing cell lysis and release of inflammatory intracellular contents. The amino acid glycine prevents pyroptotic lysis via unknown mechanisms, without affecting caspase-1 activation or pore formation. Pyroptosis plays a critical role in diverse inflammatory diseases, including sepsis. Septic lethality is prevented by glycine treatment, suggesting that glycine-mediated cytoprotection may provide therapeutic benefit. In this study, we systematically examined a panel of small molecules, structurally related to glycine, for their ability to prevent pyroptotic lysis. We found a requirement for the carboxyl group, and limited tolerance for larger amino groups and substitution of the hydrogen R group. Glycine is an agonist for the neuronal glycine receptor, which acts as a ligandgated chloride channel. The array of cytoprotective small molecules we identified resembles that of known glycine receptor modulators. However, using genetically deficient Glrb mutant macrophages, we found that the glycine receptor is not required for pyroptotic cytoprotection. Furthermore, protection against pyroptotic lysis is independent of extracellular chloride conductance, arguing against an effect mediated by ligand-gated chloride channels. Finally, we conducted a small-scale, hypothesis-driven small-molecule screen and identified unexpected ion channel modulators that prevent pyroptotic lysis with increased potency compared to glycine. Together, these findings demonstrate that pyroptotic lysis can be pharmacologically modulated and pave the way toward identification of therapeutic strategies for pathologic conditions associated with pyroptosis.
\end{abstract}

\section{Introduction}

Pyroptosis is a programmed process of lytic, proinflammatory cell death ${ }^{1}$ involved in a host of disorders including sepsis, stroke, intestinal inflammation, and Tcell depletion during HIV infection ${ }^{2-5}$. Although pyroptosis contributes to pathological inflammation and cell death, it is also an essential protective host response to infection $^{6}$. Pyroptosis is mediated by proteases in the caspase-1 family, which are activated by the innate immune signaling platforms termed inflammasomes. Inflammasomes respond to microbial or damageassociated stimuli via pattern recognition receptors in the NOD-like receptor (NLR) and AIM2-like receptor

\footnotetext{
Correspondence: Susan L. Fink (sfink@uw.edu)

'Department of Laboratory Medicine, University of Washington, Seattle, WA, USA

${ }^{2}$ Department of Microbiology, University of Washington, Seattle, WA, USA Edited by J.-E. Ricci
}

families ${ }^{7}$. Caspase- 1 proteolytically converts the proforms of interleukin $1 \beta$ (IL-1 $\beta$ ) and IL-18 to mature inflammatory cytokines. Caspase-1 also cleaves gasdermin D, releasing the $\mathrm{N}$-terminal pore-forming domain, which inserts into the plasma membrane ${ }^{8}$. Gasdermin D pores mediate osmotic cell swelling, rupture of the plasma membrane, and release of intracellular contents including the enzyme lactate dehydrogenase $(\mathrm{LDH})^{9,10}$. The importance of pyroptotic death in the pathogenesis of disease is highlighted by protection of gasdermin D knockout mice from conditions including septic lethality $^{11}$ and autoinflammatory disease $\mathrm{e}^{12,13}$.

Glycine is a simple amino acid, which prevents terminal loss of membrane integrity during pyroptosis via unknown mechanisms ${ }^{14}$. Glycine does not inhibit upstream caspase- 1 activation, pore formation, IL- $1 \beta$ secretion, or loss of mitochondrial membrane potential, but specifically affects the final lytic event, demonstrating

\section{(c) The Author(s) 2019}

(c) (i) Open Access This article is licensed under a Creative Commons Attribution 4.0 International License, which permits use, sharing, adaptation, distribution and reproduction cc) in any medium or format, as long as you give appropriate credit to the original author(s) and the source, provide a link to the Creative Commons license, and indicate if changes were made. The images or other third party material in this article are included in the article's Creative Commons license, unless indicated otherwise in a credit line to the material. If material is not included in the article's Creative Commons license and your intended use is not permitted by statutory regulation or exceeds the permitted use, you will need to obtain permission directly from the copyright holder. To view a copy of this license, visit http://creativecommons.org/licenses/by/4.0/. 
that this process can be independently manipulated ${ }^{9,15,16}$. Glycine also has a well-demonstrated cytoprotective effect on cell death resulting from hypoxia and oxidant injury (reviewed in ref. ${ }^{17}$ ). In these models, there is no role for glycine metabolism, ATP preservation, changes in cytosolic calcium, intracellular $\mathrm{pH}$ regulation, or cytoskeletal stabilization. The presence of glycine during hypoxic injury prevents loss of viability and allows cells to recover respiratory function and ATP levels upon reoxygenation $^{18}$. Although the mechanism underlying glycine protection against hypoxia and oxidant injury is incompletely understood, multiple lines of evidence point to glycine acting as a ligand at an unidentified cell surface receptor $^{17}$. Glycine administration is highly protective in models of sepsis ${ }^{19-21}$, suggesting that understanding the mechanism of glycine action may provide novel therapeutic targets for inflammasome-mediated pathology. In this study, we describe specific structural requirements for glycine protection against pyroptotic lysis. We additionally identify novel inhibitors of pyroptotic lysis with increased potency compared to glycine.

\section{Results}

\section{Structural requirements for glycine cytoprotection}

Glycine is a simple amino acid with a single carbon attached to an amino and a carboxyl group. To understand the structural requirements for cytoprotection during pyroptosis, we systematically tested a panel of amino acids and related small molecules for their ability to prevent pyroptotic lysis (Supplemental Fig. S1). We used Salmonella infection and anthrax lethal toxin to trigger pyroptosis in murine bone marrow-derived macrophages (BMDMs) via the NLRC4 and NLRP1b inflammasomes, respectively ${ }^{22}$. Pyroptotic lysis was assessed by measuring release of the large cytoplasmic enzyme, LDH. Consistent with prior studies ${ }^{14}$, we observed that glycine prevented LDH release from Salmonella-infected and lethal toxin-treated macrophages (Fig. 1a, b and Fig. S2A), without affecting the detection of $\mathrm{LDH}$ released from detergent-treated cells (Fig. S3A). We observed robust inhibition with $5 \mathrm{mM}$ glycine (Fig. S2A) and an IC50 of 1.6-1.8 mM (Table S1). Caspase-1 activation, as assessed by labeling with the caspase-1 activity probe FAM-YVAD$\mathrm{FMK}^{23}$, was not affected by glycine (Fig. 1c and Fig. S2B).

We then examined amino group substitutions, and found that $\beta$-alanine also inhibited pyroptotic lysis (Fig. 1a, b), albeit with a reduced potency compared to glycine, reaching maximal inhibition at $45 \mathrm{mM}$ (IC50 of 9.5-10.1 mM, Table S1). Similarly to glycine, $\beta$-alanine did not prevent caspase- 1 activation (Fig. 1c and Fig. S2B), or LDH release from detergent-treated cells (Fig. S3A). However, neither $\gamma$-aminobutyric acid (GABA), sarcosine, nor taurine protected against pyroptotic lysis (Fig. 1a, b). These results suggest that there may be molecular size constraints for cytoprotection, as insertion of one methylene group between the carboxyl and amino termini is tolerated ( $\beta$-alanine), while two are not (GABA). Substitution of the amino group with a simple hydrogen (acetate) or methyl group (propionate) afforded protection against pyroptotic lysis only at higher concentrations (Fig. 1d, e), which did not affect detection of LDH released from detergent-treated cells (Fig. S3B), suggesting that the amino group contributes to protection, but may not be absolutely required.

We then examined other amino acids with $\mathrm{R}$ group substitutions, and found inhibition of pyroptotic lysis by both alanine enantiomers, as well as the cyclic amino acid, 1aminocyclopropane carboxylic acid (1-ACPC) (Fig. 2a, b and Table S1), without an effect on caspase-1 activation (Fig. 2c) or detergent-mediated LDH release (Fig. S3C). D-serine demonstrated modest protection at higher concentrations, while $\mathrm{L}$-serine and $\mathrm{L}$ - and $\mathrm{D}$-valine were without protective effect (Fig. 2a, b). These results suggest that specific substitutions of the hydrogen $\mathrm{R}$ group maintain cytoprotective effects, but with lower potency compared to glycine.

Finally, we examined carboxyl substitutions, and found no inhibition of Salmonella-induced pyroptotic lysis with methylamine, ethylamine, or ethanolamine (Fig. 3a). Methylamine and ethylamine prevent endosome acidification, which is required for anthrax lethal toxin entry ${ }^{24}$ and subsequent induction of pyroptosis (Fig. S4). However, ethanolamine was not protective against lethal toxin-induced pyroptotic lysis (Fig. 3b). These results indicate that the carboxyl group is absolutely required for cytoprotection.

Together, these results reveal a subset of molecules, structurally related to glycine, that provide protection against pyroptotic lysis, with a decreasing potency order of: glycine $>\beta$-alanine, 1-ACPC, L-alanine $>$ D-alanine. These findings define specific structural requirements for small molecule-mediated protection against pyroptotic lysis.

\section{GlyR modulators prevent pyroptotic lysis, but glycine cytoprotection is independent of GlyR}

Glycine is well known to bind the glycine receptor (GlyR) and act as an inhibitory neurotransmitter ${ }^{25}$. Ligand binding triggers opening of the GlyR chloride channel, allowing chloride influx to hyperpolarize the neuronal membrane and limit depolarizing signals. Glycine is the classical agonist for GlyR, but other amino acids also have activity with a potency order of: glycine $>\beta$-alanine $>$ L-alanine $>$ D-alanine, while GABA fails to activate $G l y R^{26,27}$. This pattern of activity is remarkably similar to our observations for protection against pyroptotic lysis, suggesting the hypothesis that glycine cytoprotection is mediated by GlyR.

GlyR is a pentameric receptor composed of alpha and beta subunits; four differentially expressed alpha subunits and one beta subunit have been identified ${ }^{28}$. The beta subunit interacts with the scaffolding protein gephyrin, 

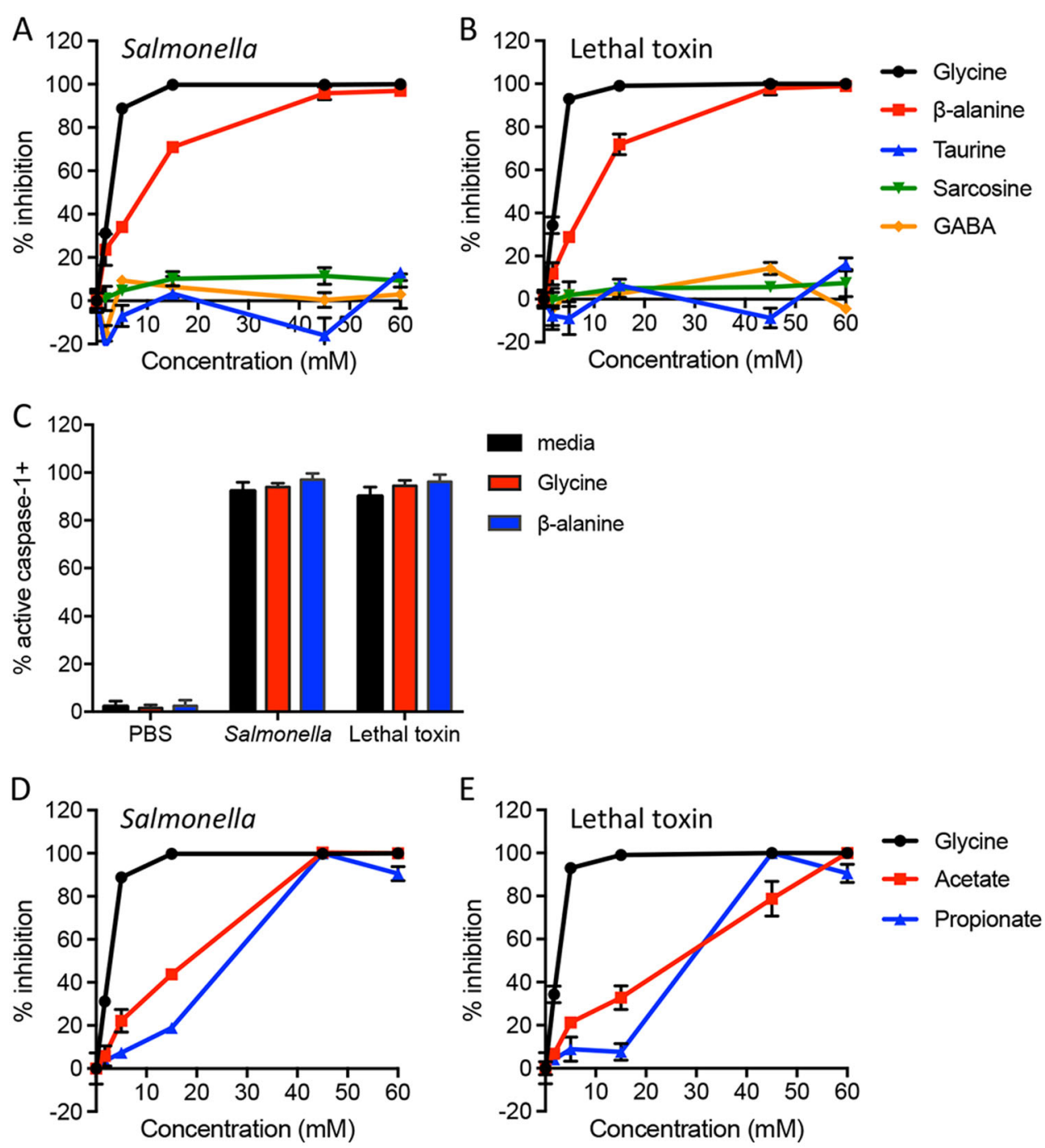

Fig. 1 Specific small molecules with structural similarity to glycine inhibit pyroptotic lysis. Bone marrow-derived macrophages were treated with Salmonella $(\mathbf{a}, \mathbf{d})$ or anthrax lethal toxin $(\mathbf{b}, \mathbf{e})$ in the presence of glycine or related small molecules that vary at the amino position (titrated from 1.7-60 mM). LDH released during pyroptotic lysis was measured and compared to LDH released in the absence of inhibitor (\% inhibition). Representative data (mean $\pm S D, n=3$ ) from three or more independent experiments are shown. $\mathbf{c}$ To determine whether glycine or $\beta$-alanine block caspase-1 activation, macrophages were treated with PBS, Salmonella or anthrax lethal toxin in the presence of medium alone, $5 \mathrm{mM}$ glycine or $15 \mathrm{mM} \beta$-alanine. Active caspase-1 was identified by FAM-YVAD-FMK staining. Cumulative data from two independent experiments (mean \pm SD, $n=$ 7 high power fields with 328-574 total cells queried per condition) are shown

and heteropentameric receptors are thought to consist of either two alpha and three beta subunits or three alpha and two beta subunits ${ }^{29-31}$. In heterologous expression systems, alpha subunits alone are sufficient to produce functional homomeric glycine-gated channels ${ }^{28}$. Alpha subunit expression is anatomically and developmentally regulated in the nervous system. Expression of GlyR subunits in nonneuronal cell types has been described, and functional evidence suggests the possibility of GlyR activity in macrophages ${ }^{32,33}$.

Propofol is an anesthetic that potentiates the GlyR and $500 \mu \mathrm{M}$ propofol allosterically enhances GlyR responses to the partial agonists, $\beta$-alanine, and taurine $^{34}$. We therefore tested the hypothesis that propofol might potentiate the cytoprotective ability of $\beta$-alanine and taurine against pyroptotic lysis. Propofol alone afforded no protection against pyroptotic lysis, but enhanced the cytoprotective ability of $\beta$-alanine (Fig. 4a). Additionally, taurine alone had no cytoprotective effect (Fig. 1a), but in combination with $150 \mu \mathrm{M}$ propofol, we observed partial inhibition of pyroptotic lysis (Fig. 4a). Together, these results suggest that protection from pyroptotic lysis mirrors the agonist properties of ligands at the GlyR. 

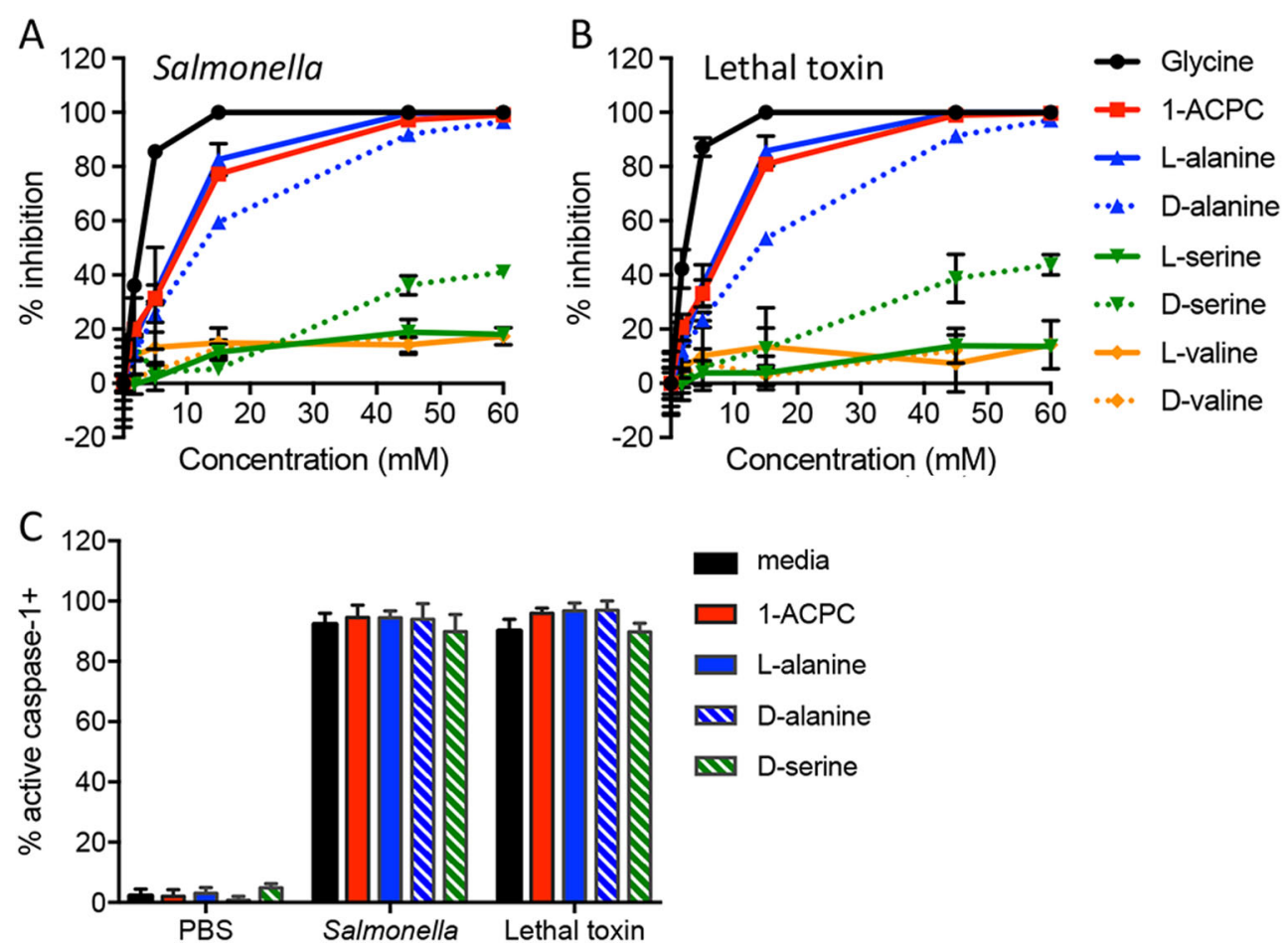

Fig. 2 Substitution of amino acid side chains influences cytoprotection. Bone marrow-derived macrophages were treated with Salmonella (a) or anthrax lethal toxin $(\mathbf{b})$ in the presence of glycine or related amino acids with $\mathrm{R}$ group substitutions (titrated from 1.7-60 mM). LDH released during pyroptotic lysis was measured and compared to LDH released in the absence of inhibitor (\% inhibition). Representative data (mean $\pm S D, n=3$ ) from three or more independent experiments are shown. c Cytoprotective amino acids do not block caspase-1 activation. Macrophages were treated with PBS, Salmonella or anthrax lethal toxin in the presence of medium alone, glycine (5 mM), 1-aminocyclopropane carboxylic acid (1-ACPC, 15 mM), Lalanine $(15 \mathrm{mM})$, D-alanine $(45 \mathrm{mM})$, or D-serine $(15 \mathrm{mM})$. Active caspase-1 was identified by FAM-YVAD-FMK staining. Cumulative data from two independent experiments (mean \pm SD, $n=7$ high power fields with 357-588 total cells queried per condition) are shown

Strychnine (Fig. S5) is the classical GlyR antagonist and blocks glycine-mediated effects at this receptor at nanomolar concentrations ${ }^{35}$. We did not observe antagonism of glycine cytoprotection by strychnine (Fig. S6A), but surprisingly found that strychnine alone mimicked glycine cytoprotection (Fig. S6A and Fig. 4b). Strychnine prevented pyroptotic lysis with increased potency compared to glycine, with an IC50 of $0.5-0.8 \mathrm{mM}$ (Table S1), without affecting caspase-1 activation (Fig. 4c) or detection of LDH released from detergent-treated cells (Fig. S6B). Although strychnine is classically a GlyR antagonist with affinity in the $5-15 \mathrm{nM}$ range $\mathrm{e}^{35}$, at higher concentrations strychnine can act as an agonist for glycinegated chloride channels ${ }^{36,37}$. This could potentially explain our observation that strychnine alone provided cytoprotection and synergistically potentiated cytoprotection in presence of glycine. We then examined brucine (Fig. S5), another well-characterized GlyR antagonist ${ }^{38}$. We found that brucine mimicked glycine and strychnine in preventing lysis during pyroptosis without affecting caspase-1 activation or detection of LDH released from detergent-treated cells (Fig. 4b, c and Fig. S6B). The IC50 of brucine $(0.6-0.7 \mathrm{mM})$ was similar to strychnine.
Notably, these are much higher than the nanomolar concentrations needed for inhibition at the GlyR.

These results were consistent with the hypothesis that the GlyR is involved in protection against pyroptotic lysis. To genetically determine whether the GlyR is required for the protective effect of glycine, we cultured macrophages from homozygous spastic (spa) mutant mice. The spa mutation is a 7 kilobase insertion in intron 6 of Glrb, causing aberrant splicing and reducing expression of the GlyR beta subunit to undetectable levels ${ }^{27,39}$. Macrophages from spa/spa mice underwent pyroptotic lysis that was inhibited by both glycine and strychnine (Fig. 5a), suggesting that canonical heteromeric GlyRs containing the beta subunit are dispensable for cytoprotection.

The neuronal GlyR is a heteropentamer comprised of alpha and beta subunits, but in heterologous expression systems, alpha subunits alone are sufficient to produce functional homomeric glycine-gated channels ${ }^{28}$. Although spa mutant macrophages lacking the beta subunit exhibit glycine cytoprotection, this does not exclude the possibility of homomeric alpha subunit GlyR channels mediating protection against pyroptotic lysis. Both homo- and hetero-oligomeric GlyR channels share strong selectivity 

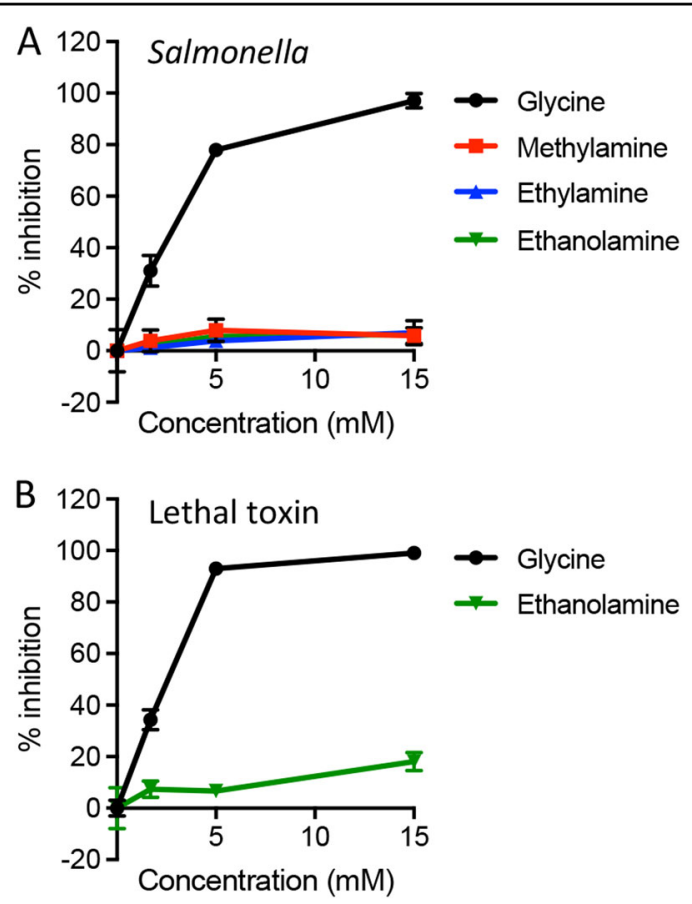

Fig. 3 The carboxyl group is essential for protecting cells from pyroptotic lysis. a Bone marrow-derived macrophages were infected with Salmonella in the presence of glycine, methylamine, ethylamine, or ethanolamine (titrated from 1.7-15 mM). b Macrophages were treated with anthrax lethal toxin in the presence of glycine or ethanolamine. LDH released during pyroptotic lysis was measured and compared to $L D H$ released in the absence of inhibitor (\% inhibition). Representative data (mean $\pm \mathrm{SD}, n=3$ ) from three or more independent experiments are shown

for permeating chloride ions and do not conduct larger gluconate ions ${ }^{40}$. To determine whether glycine protection against pyroptotic lysis requires extracellular chloride conductance, we treated macrophages in medium in which chloride was replaced with larger gluconate anions. We found that macrophages underwent pyroptotic lysis that was blocked by glycine when cultured in either chloride or gluconate-containing medium (Fig. 5b). These findings suggest that glycine protection against pyroptotic lysis does not require extracellular chloride conductance, arguing against an effect mediated by either homo- or hetero-oligomeric GlyR channels. Together, these data refute the implied correlation between observed potency order (glycine $>\beta$-alanine $>$ L-alanine $>$ D-alanine) and GlyR function and suggest that these small molecules act at another target to prevent pyroptotic lysis.

\section{Identification of novel inhibitors of pyroptotic lysis}

Strychnine and brucine are specific for GlyR when used at nanomolar concentrations ${ }^{35}$, but protection from pyroptotic lysis requires micromolar concentrations, potentially consistent with lower affinity binding to a related
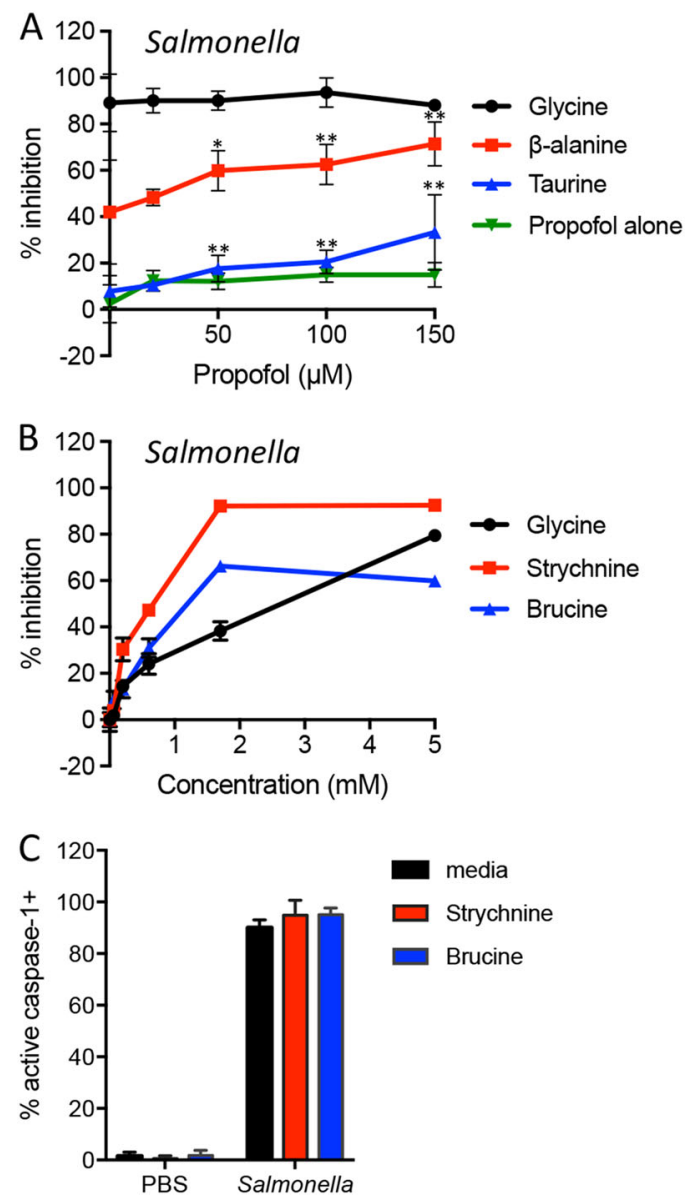

Fig. 4 Glycine receptor agonists and antagonists both inhibit pyroptotic lysis. a Macrophages were pretreated with increasing concentrations of propofol for $30 \mathrm{~min}$ prior to addition of the GlyR agonists, glycine, $\beta$-alanine, or taurine (all $5 \mathrm{mM}$ ). Salmonella-induced $L D H$ release was measured and compared to $L D H$ released by pyroptotic cells in the absence of all inhibitors (\% inhibition). Representative data (mean $\pm \mathrm{SD}, n=3$ ) from three or more independent experiments are shown. ${ }^{*} P<0.05,{ }^{* *} P<0.01$ (unpaired t-test) indicates significance compared to $0 \mathrm{mM}$ propofol. $\mathbf{b}$ Macrophages were infected with Salmonella in the presence of GlyR antagonists, strychnine, or brucine (titrated from 0.06 to $5 \mathrm{mM}$ ). Representative data (mean $\pm S D, n=3$ ) from three or more independent experiments are shown. c GlyR antagonists do not block caspase-1 activation. Macrophages were treated with PBS or

Salmonella in the presence of medium alone, $1.7 \mathrm{mM}$ strychnine, or $1.7 \mathrm{mM}$ brucine. Active caspase-1 was identified by FAM-YVAD-FMK staining. Cumulative data from two independent experiments (mean $\pm \mathrm{SD}, n=7$ high power fields with 413-578 total cells queried per condition) are shown

receptor. To identify novel inhibitors of pyroptotic lysis, we performed a small-scale, hypothesis-driven screen of small molecules with known activities at the GlyR and related ligand-gated ion channels (Fig. S5 and Table 1).

As we observed inhibition of pyroptotic lysis by the GlyR antagonists strychnine and brucine, we first sought to determine whether other GlyR antagonists prevent 


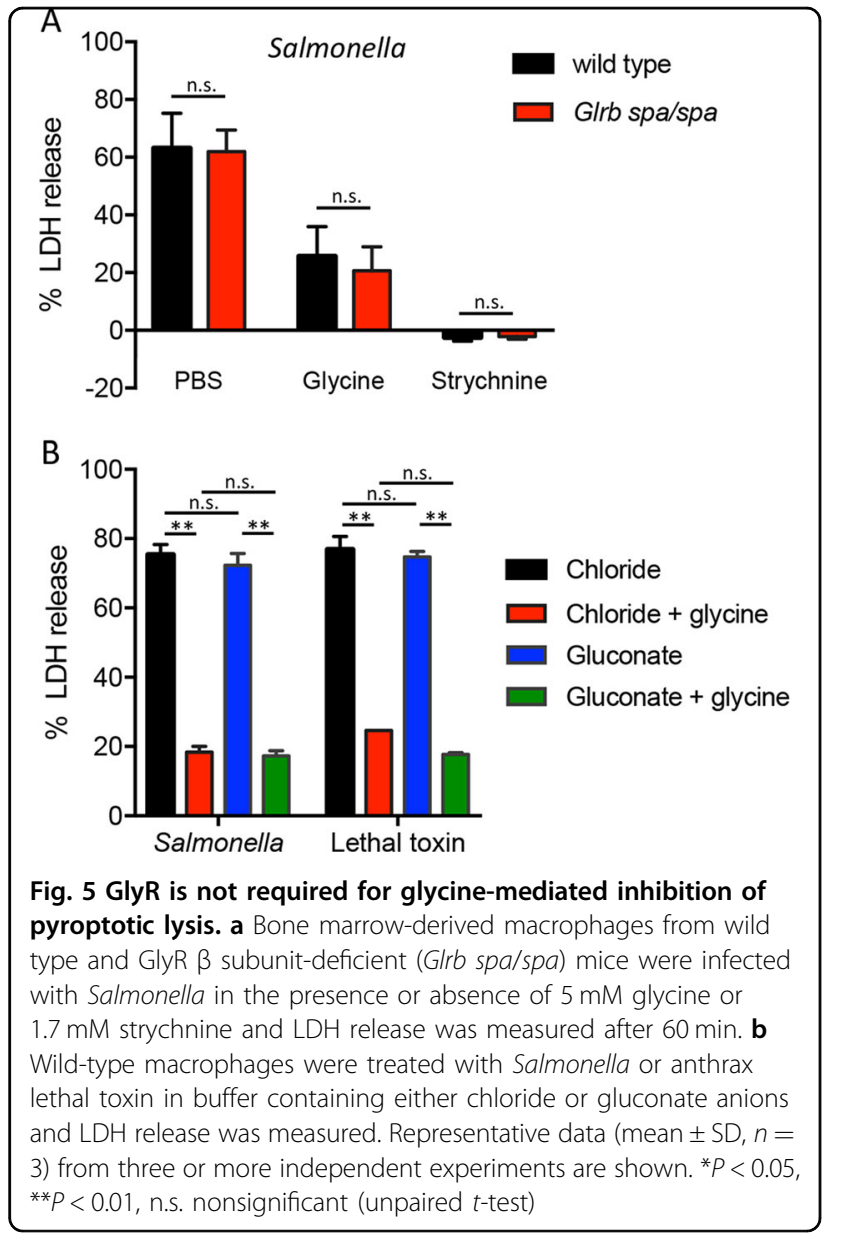

pyroptotic lysis. Nipecotic acid is a competitive GlyR antagonist like strychnine and brucine ${ }^{26}$. However, unlike strychnine and brucine, nipecotic acid did not protect against pyroptotic lysis (Fig. 6a, b). In addition, nipecotic acid failed to antagonize the protective effect of glycine and strychnine (Fig. S7). These results suggest that some, but not all, GlyR modulators prevent pyroptotic lysis, consistent with our findings that protection does not require the hetero-oligomeric GlyR channel.

Pregnenolone sulfate is a neurosteroid that inhibits $\mathrm{GlyR}^{41}$, but also blocks $\mathrm{GABA}_{\mathrm{A}}$ receptors ${ }^{42}$. Pregnenolone sulfate potently inhibited pyroptotic lysis induced by both Salmonella and lethal toxin (Fig. 6c, d), with an IC50 of 0.06-0.07 mM (Table S1). Pregnenolone sulfate did not affect caspase-1 activation (Fig. 6e) or detection of LDH from detergent-treated cells (Fig. S8B). We then examined other inhibitors of both GlyR and $\mathrm{GABA}_{\mathrm{A}}$ receptors. We found no significant protection against pyroptotic lysis with the prototypic $\mathrm{GABA}_{\mathrm{A}}$ receptor antagonist, picrotoxin, which also blocks homomeric GlyR receptors ${ }^{35}$ (Fig. 6a, b). Ginkgolide B also inhibits both $\mathrm{GlyR}^{43}$ and $\mathrm{GABA}_{\mathrm{A}}$ receptors ${ }^{44}$, but was not significantly protective against pyroptotic lysis (Fig. 6a, b).
Table 1 A subset of ion channel modulators prevent pyroptotic lysis

\begin{tabular}{llll}
\hline & $\begin{array}{l}\text { Inhibits } \\
\text { pyroptotic lysis }\end{array}$ & GlyR & GABA $_{\mathbf{A}}$ \\
\hline Strychnine & Yes & Antagonist/ & \\
Brucine & Yes & agonist & \\
Nipecotic acid & No & Antagonist/ & \\
Pregnenolone & Yes & agonist & \\
sulfate & & Antagonist & Antagonist \\
Picrotoxin & No & Antagonist & Antagonist \\
Ginkgolide B & No & Antagonist & Antagonist \\
Bicuculline & No & Antagonist & Antagonist \\
Muscimol & Yes & & Agonist \\
THIP & No & & Agonist \\
\hline
\end{tabular}

Given that pregnenolone sulfate acts at $\mathrm{GABA}_{\mathrm{A}}$ receptors, we then examined additional $\mathrm{GABA}_{\mathrm{A}}$ receptor modulators. We found no protection against pyroptotic lysis with bicuculline (Fig. 6f, g), a selective $\mathrm{GABA}_{\mathrm{A}}$ receptor antagonist ${ }^{35}$. However, the classic GABA $_{A}$ receptor agonist, muscimol, potently inhibited pyroptotic lysis induced by both Salmonella and anthrax lethal toxin, with an IC50 of $0.3 \mathrm{mM}$ (Fig. 6f, g and Table S1). Muscimol did not affect inflammasome-induced caspase-1 activation or detection of $\mathrm{LDH}$ from detergent-treated cells (Fig. $6 \mathrm{~h}$ and Fig. S8C). In contrast, the conformationally restrained muscimol analog, 4,5,6,7-tetrahydroisoxazolo(5,4-c)pyridin-3-ol (THIP) ${ }^{45}$, did not significantly protect against pyroptotic lysis (Fig. 6f, g). As the protective effect of muscimol is not recapitulated by another $\mathrm{GABA}_{\mathrm{A}}$ agonist, this curious inconsistency suggests that the $\mathrm{GABA}_{\mathrm{A}}$ receptor may not mediate muscimol protection. Together, these results reveal a novel panel of diverse small molecules with unexpected potent activity to protect against pyroptotic lysis.

\section{Discussion}

Glycine protection from pyroptotic lysis has long been recognized, but this study is the first to examine the molecular requirements for this effect. Osmoprotectants, large inert molecules that cannot pass through plasma membrane pores, block pyroptotic lysis by balancing osmotic differences between the cytosol and the extracellular milieu. Osmoprotectants were initially used to predict the existence of a caspase-1-dependent plasma membrane pore, prior to the identification of gasdermin $\mathrm{D}^{9}$. Glycine has been referred to as an osmoprotectant, 


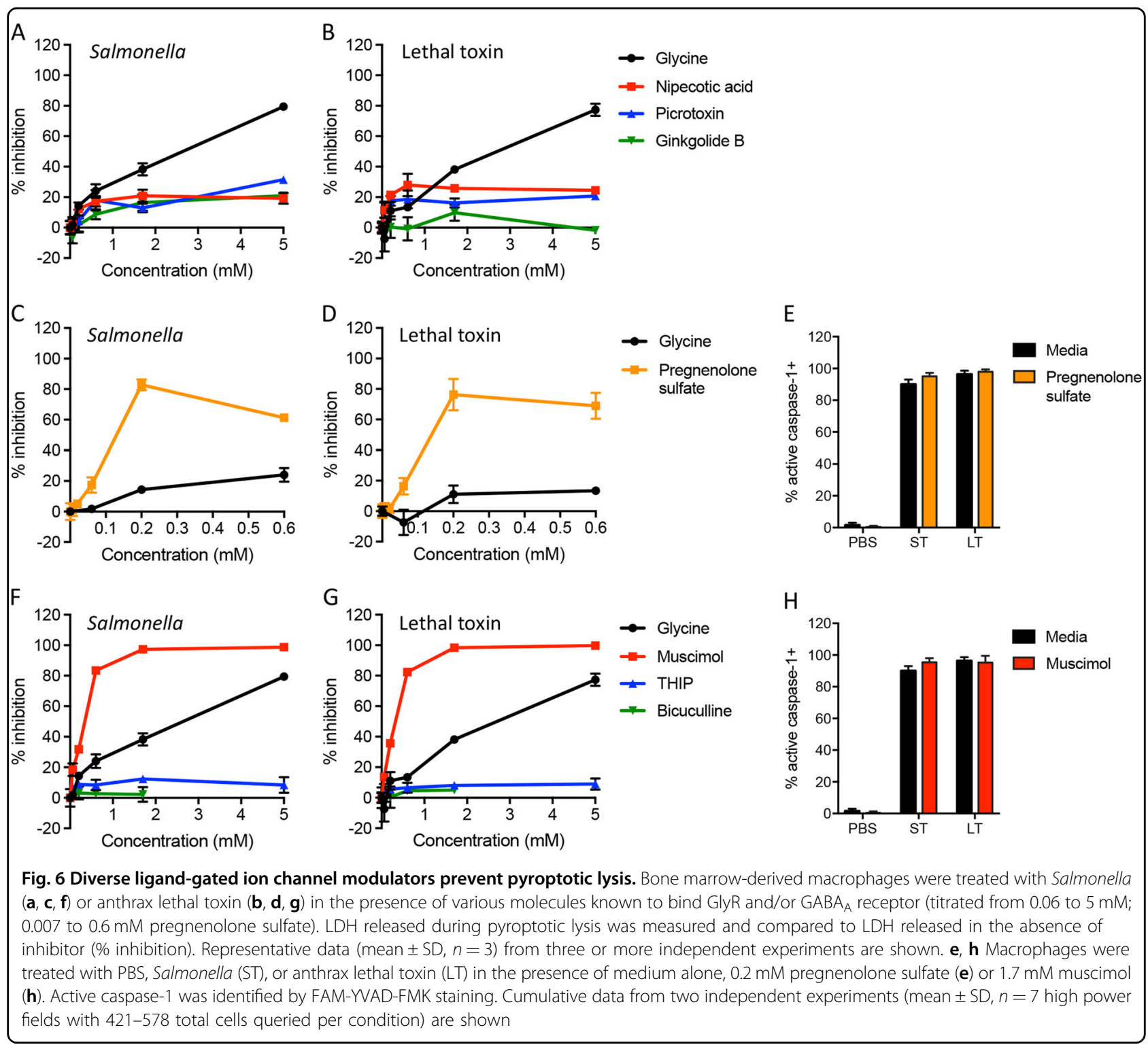

however its small size would allow free passage through the large gasdermin $\mathrm{D}$ pore, preventing glycine from being able to balance the osmotic forces. Indeed, other similarly sized molecules, such as taurine, serine and ethanolamine do not protect against pyroptotic lysis (Figs. 1-3). Together, our data reveal specific structural requirements for protection, which may be mediated by an unidentified receptor. Alternatively, glycine and structurally similar small molecules may exert a cytoprotective effect by other mechanisms, such as modulating the regulatory volume decrease, which would be expected to engage during pyroptotic cell swelling.

Glycine cytoprotection has been well documented in diverse cell types in other models of cell death, including ATP depletion and oxidant injury (reviewed in ref. ${ }^{17}$ ). In these models, glycine protection is reversible and there is no role for glycine metabolism, ATP preservation, intracellular $\mathrm{pH}$ regulation, or cytoskeletal stabilization. During pyroptosis, glycine protection is also rapidly reversible ${ }^{15}$ and cellular ATP is not preserved ${ }^{16}$. The profile of molecules that protect against ATP depletion and oxidant injury does not match known GlyRs, but does share some similarities with our findings. For example, similarly to pyroptosis, $\beta$ alanine is protective against hypoxic death, while taurine is not $^{46}$. Strychnine also protects against ATP depletion and oxidant injury, and acts at the cell surface ${ }^{47-49}$. More limited data also suggests cytoprotection against hypoxia with pregnenolone sulfate and muscimol ${ }^{50}$. However, in contrast to our findings with pyroptosis, bicuculline also protects against hypoxic cell death ${ }^{47,48}$.

In addition to glycine, we found other amino acids that afford protection against pyroptotic lysis, with a potency 
order of glycine $>\beta$-alanine, L-alanine $>$ D-alanine. This pattern curiously resembles that of known GlyR agonists. However, taurine is an additional well-described GlyR agonist $^{25}$ and afforded no cytoprotection alone (Figs. 1a, b and 4a). Furthermore, D-serine and 1-ACPC protect against pyroptotic lysis but have no agonist activity at $\mathrm{GlyR}^{26,27,51}$. Further evidence against a role for GlyR in cytoprotection is the dispensability of the GlyR beta subunit, which is necessary for formation of heteromeric GlyR. Finally, replacement of chloride with gluconate excludes a requirement for extracellular chloride conductance, which is the major function of $\mathrm{GlyR}^{28}$. The requirement for the GlyR in glycine protection against hypoxia and oxidant injury has been inconsistent, with some models requiring the GlyR, but most not ${ }^{17}$. Therefore, cytoprotection in different models of cellular injury may not share a common mechanism, but in the case of glycine protection against pyroptotic lysis, we show that GlyR is not required.

In addition to structurally similar small molecules, this study has identified novel cytoprotective compounds with increased potency compared to glycine. These include the canonical GlyR antagonists strychnine and brucine (Fig. 4), the modulatory neurosteroid pregnenolone sulfate, and the canonical $\mathrm{GABA}_{\mathrm{A}}$ receptor agonist, muscimol (Fig. 6). None of these inhibitors affected caspase-1 activation, indicating that they specifically block pyroptotic lysis downstream of inflammasome activation. All of these are more potent than glycine, but still require concentrations higher than is typically needed for activity at their canonical receptors. The profile of cytoprotection also does not correspond with the classic receptors targeted by these agents, as neither the GlyR inhibitor nipecotic acid nor the $\mathrm{GABA}_{\mathrm{A}}$ receptor agonists THIP or GABA itself provided protection. Additionally, the $\mathrm{GABA}_{\mathrm{A}}$ receptor selectively conducts chloride ${ }^{52}$, which is dispensable for protection from pyroptosis, and argues against a role of the $\mathrm{GABA}_{\mathrm{A}}$ receptor. The GlyR and $\mathrm{GABA}_{\mathrm{A}}$ receptors are related ligand-gated ion channels, with a number of shared features, including potentiation by propofol ${ }^{53}$. Small molecules with diverse structures act as allosteric modulators of these receptors and alter receptor activity by interacting with binding sites distinct from the primary ligand-binding site ${ }^{54,55}$. We hypothesize that the cytoprotective ability of some GlyR and $\mathrm{GABA}_{\mathrm{A}}$ modulators may result from modulation of an unidentified receptor with similarity to the GlyR and $G_{A B A}$ receptors. As glycine does not prevent gasdermin Dmediated pore formation ${ }^{9,16,56}$, we hypothesize that glycine and other cytoprotectants do not bind gasdermin D directly, but modulate lysis in conjunction with an additional unidentified protein.

During pyroptosis, glycine prevents rapid membrane disruption, but not IL-1 $\beta$ secretion, pore-mediated small dye uptake, loss of ATP, or induction of secondary cell death pathways; thus, glycine-treated cells do not remain intact indefinitely ${ }^{9,16,56,57}$. Preventing rapid membrane disruption may not ultimately rescue cells from lethality ${ }^{15}$, but may impact the physiologic consequences of cell death by preventing rapid exposure of immunostimulatory intracellular contents, such as actin ${ }^{58,59}$ and mitochondria $^{60}$. Pyroptotic lysis also releases free inflammasomes, which propagate inflammatory responses in bystander cells ${ }^{61,62}$. In addition to promoting inflammation, membrane breakdown may expose "eat-me" signals that promote efferocytosis of pyroptotic cells and clearance of trapped pathogens ${ }^{63}$. Therefore, mechanisms that regulate terminal lysis during pyroptosis may contribute to the inflammatory consequences of this form of cell death, as well as pyroptotic control of infection.

Sepsis is a common and highly fatal condition associated with pyroptosis ${ }^{2}$. In mouse models, septic lethality is mediated by gasdermin $\mathrm{D}$, demonstrating the fatal consequences of rapid pyroptotic lysis ${ }^{11,64}$. Glycine administration is highly protective in models of sepsis ${ }^{19-21}$, suggesting that understanding the mechanism of glycine action may provide novel therapeutic targets for inflammasome-mediated pathology. This study identified novel pyroptotic cytoprotectants of much higher potency than glycine, which may be useful for future studies of pyroptosis.

\section{Materials and methods \\ Cell culture}

BMDMs were cultured from wild-type Balb/c mice (Jackson Labs) for 7 days at $37^{\circ} \mathrm{C}$ in $5 \% \mathrm{CO}_{2}$ in DMEM supplemented with $10 \%$ FCS, $5 \mathrm{mM}$ HEPES, $0.2 \mathrm{mg} / \mathrm{ml} \mathrm{L}-$ glutamine, $0.05 \mathrm{mM} \beta$-mercaptoethanol, $50 \mu \mathrm{g} / \mathrm{ml}$ gentamicin sulfate, $100 \mathrm{U} / \mathrm{ml}$ penicillin and streptomycin, and 30\% L-cell-conditioned medium. Macrophages were collected by washing with ice-cold PBS containing $1 \mathrm{mM}$ EDTA and resuspended in supplemented antibiotic-free DMEM (without phenol red) containing 5\% FCS (DMEM-5). For the GlyR $\beta$ subunit experiment, BMDM were cultured from wild-type C57BL/6 and B6.CgGlrb $^{\text {spa }} /$ J mice (JAX \#000066) (Jackson Labs). To test the importance of GlyR chloride channel function, macrophages were washed with and incubated in $\mathrm{NaCl}-\mathrm{HBSS}$ or gluconate-HBSS: $138 \mathrm{mM} \mathrm{NaCl}$ (or Na gluconate), $5 \mathrm{mM}$ $\mathrm{KCl}$ (or $\mathrm{K}$ gluconate), $1.8 \mathrm{mM} \mathrm{CaCl}_{2}$ (or Ca gluconate), $4.2 \mathrm{mM} \mathrm{NaHCO}_{3}, 0.34 \mathrm{mM} \mathrm{NaH}_{2} \mathrm{PO}_{4}-\mathrm{H}_{2} \mathrm{O}$, and $0.44 \mathrm{mM}$ $\mathrm{KH}_{2} \mathrm{PO}_{4}$.

\section{Lactate dehydrogenase (LDH) assay}

Macrophages were pretreated for $30 \mathrm{~min}$ with glycine or one of the other small-molecule inhibitors at the indicated dose. All chemicals were purchased from Sigma-Aldrich, with the exception of propofol (EMD Millipore). Late-log 
cultures of Salmonella typhimurium SL1344 grown in Lbroth containing $0.3 \mathrm{M} \mathrm{NaCl}$ were used to infect macrophages (MOI 10:1) for $90 \mathrm{~min}$, unless otherwise indicated. Cells were treated with anthrax lethal toxin comprised of $1 \mu \mathrm{g} / \mathrm{ml}$ protective antigen and $1 \mu \mathrm{g} / \mathrm{ml}$ lethal factor (List Biological) for $2 \mathrm{~h}^{14}$. Cells were treated with the detergent-based lysis buffer included in the Cytotox 96 Kit (Promega) for $30 \mathrm{~min}$. Release of cytoplasmic LDH was determined from triplicate samples using the Cytotox 96 Kit and calculated as 100 (experimental LDH - spontaneous LDH)/(maximum LDH -spontaneous LDH).

\section{Fluorescence microscopy}

Macrophages were seeded onto glass coverslips at a density of $2 \times 10^{5}$ cells/well (24-well plate) prior to infection with S. typhimurium or treatment with anthrax lethal toxin. Active caspase-1 was detected using a caspase-1-specific probe ${ }^{23}$. FAM-YVAD-FMK (Immunochemistry Technologies) was added to a final concentration of $5 \mu \mathrm{M}$ for the last $60 \mathrm{~min}$ of treatment. Cells were washed twice to remove unbound probe, then fixed and stained with the nuclear dye To-Pro-3 (Molecular Probes). Multiple fields were examined using a Leica SL confocal microscope (W.M. Keck Microscopy Center), and the percent of positive cells was determined using 3-10 fields for each experimental condition.

\section{Statistics}

Data were analyzed by unpaired two-tailed Student's $t$ test. IC50 values were determined using nonlinear regression and the [Inhibitor] vs. response - variable slope equation in Prism software (GraphPad).

\section{Acknowledgments}

We thank Matthew Johnson for technical assistance. S.L.F. was supported by the National Institute of Allergy and Infectious Diseases of the National Institutes of Health under award number K08Al119142. The content is solely the responsibility of the authors and does not necessarily represent the official views of the National Institutes of Health.

\section{Conflict of interest}

The authors declare that they have no conflict of interest.

\section{Publisher's note}

Springer Nature remains neutral with regard to jurisdictional claims in published maps and institutional affiliations.

Supplementary Information accompanies this paper at (https://doi.org/ 10.1038/s41419-019-1559-4).

Received: 7 January 2019 Revised: 28 March 2019 Accepted: 29 March 2019 Published online: 11 April 2019

\footnotetext{
References

1. Fink, S. L. \& Cookson, B. T. Apoptosis, pyroptosis, and necrosis: mechanistic description of dead and dying eukaryotic cells. Infect. Immun. 73, 1907-1916 (2005).
}

2. Croker, B. A., O'Donnell, J. A. \& Gerlic, M. Pyroptotic death storms and cytopenia. Curr. Opin. Immunol. 26, 128-137 (2014).

3. Sharma, D. \& Kanneganti, T. D. Inflammatory cell death in intestinal pathologies. Immunol. Rev. 280, 57-73 (2017).

4. Barrington, J., Lemarchand, E. \& Allan, S. M. A brain in flame; do inflammasomes and pyroptosis influence stroke pathology? Brain Pathol. 27, 205-212 (2017).

5. Doitsh, G. \& Greene, W. C. Dissecting how CD4 T cells are lost during HIV infection. Cell Host Microbe 19, 280-291 (2016).

6. Jorgensen, I. \& Miao, E. A. Pyroptotic cell death defends against intracellular pathogens. Immunol. Rev. 265, 130-142 (2015).

7. Martinon, F., Mayor, A. \& Tschopp, J. The inflammasomes: guardians of the body. Annu. Rev. Immunol. 27, 229-265 (2009).

8. Kovacs, S. B. \& Miao, E. A. Gasdermins: effectors of pyroptosis. Trends Cell Biol. 27, 673-684 (2017).

9. Fink, S. L. \& Cookson, B. T. Caspase-1-dependent pore formation during pyroptosis leads to osmotic lysis of infected host macrophages. Cell. Microbiol. 8, 1812-1825 (2006)

10. de Vasconcelos, N. M., Van Opdenbosch, N., Van Gorp, H., Parthoens, E. \& Lamkanfi, M. Single-cell analysis of pyroptosis dynamics reveals conserved GSDMD-mediated subcellular events that precede plasma membrane rupture. Cell Death Differ. 26, 146-161 (2019).

11. Kayagaki, N. et al. Caspase-11 cleaves gasdermin D for non-canonical inflammasome signalling. Nature 526, 666-671 (2015).

12. Xiao, J. et al. Gasdermin D mediates the pathogenesis of neonatalonset multisystem inflammatory disease in mice. PLOS Biol. 16, e3000047 (2018).

13. Kanneganti, A. et al. GSDMD is critical for autoinflammatory pathology in a mouse model of Familial Mediterranean Fever. J. Exp. Med. 215, 1519-1529 (2018).

14. Fink, S. L., Bergsbaken, T. \& Cookson, B. T. Anthrax lethal toxin and Salmonella elicit the common cell death pathway of caspase-1-dependent pyroptosis via distinct mechanisms. Proc. Natl Acad. Sci. USA 105, 4312-4317 (2008).

15. DiPeso, L., Ji, D. X., Vance, R. E. \& Price, J. V. Cell death and cell lysis are separable events during pyroptosis. Cell Death Discov. 3, 17070 (2017).

16. Russo, H. M. et al. Active caspase-1 induces plasma membrane pores that precede pyroptotic lysis and are blocked by lanthanides. J. Immunol. 197, 1353-1367 (2016).

17. Weinberg, J. M., Bienholz, A. \& Venkatachalam, M. A. The role of glycine in regulated cell death. Cell. Mol. Life Sci. 73, 2285-2308 (2016).

18. Weinberg, J. M., Davis, J. A., Abarzua, M. \& Rajan, T. Cytoprotective effects of glycine and glutathione against hypoxic injury to renal tubules. J. Clin. Invest. 80, 1446-1454 (1987).

19. Ikejima, K., limuro, Y., Forman, D. T. \& Thurman, R. G. A diet containing glycine improves survival in endotoxin shock in the rat. Am. J. Physiol. 271, G97-G103 (1996).

20. Hamburger, T. et al. Effects of glycine, pyruvate, resveratrol, and nitrite on tissue injury and cytokine response in endotoxemic rats. J. Surg. Res. 183 e7-e21 (2013).

21. Yang, S., Koo, D. J., Chaudry, I. H. \& Wang, P. Glycine attenuates hepatocellular depression during early sepsis and reduces sepsis-induced mortality. Crit. Care Med. 29, 1201-1206 (2001).

22. den Hartigh, A. B. \& Fink, S. L. Pyroptosis induction and detection. Curr. Protoc. Immunol. 122, e52 (2018).

23. den Hartigh, A. B. \& Fink, S. L. Detection of inflammasome activation and pyroptotic cell death in murine bone marrow-derived macrophages. J. Vis. Exp. e57463, https:/doi.org/10.3791/57463 (2018).

24. Friedlander, A. M. Macrophages are sensitive to anthrax lethal toxin through an acid-dependent process. J. Biol. Chem. 261, 7123-7126 (1986).

25. Betz, H. \& Laube, B. Glycine receptors: recent insights into their structural organization and functional diversity. J. Neurochem. 97, 1600-1610 (2006).

26. Schmieden, V. \& Betz, H. Pharmacology of the inhibitory glycine receptor: agonist and antagonist actions of amino acids and piperidine carboxylic acid compounds. Mol. Pharmacol. 48, 919-927 (1995).

27. Tokutomi, N., Kaneda, M. \& Akaike, N. What confers specificity on glycine for its receptor site? Br. J. Pharmacol. 97, 353-360 (1989).

28. Dutertre, S., Becker, C. M. \& Betz, H. Inhibitory glycine receptors: an update. J. Biol. Chem. 287, 40216-40223 (2012).

29. Grudzinska, J. et al. The beta subunit determines the ligand binding properties of synaptic glycine receptors. Neuron 45, 727-739 (2005). 
30. Patrizio, A., Renner, M., Pizzarelli, R., Triller, A. \& Specht, C. G. Alpha subunitdependent glycine receptor clustering and regulation of synaptic receptor numbers. Sci. Rep. 7, 10899 (2017).

31. Kasaragod, V. B. \& Schindelin, H. Structure-function relationships of glycine and GABAA receptors and their interplay with the scaffolding protein gephyrin. Front. Mol. Neurosci. 11, 317 (2018).

32. Froh, M., Thurman, R. G. \& Wheeler, M. D. Molecular evidence for a glycinegated chloride channel in macrophages and leukocytes. Am. J. Physiol. Gastrointest. Liver Physiol. 283, G856-G863 (2002).

33. Wheeler, M. D. \& Thurman, R. G. Production of superoxide and TNF-alpha from alveolar macrophages is blunted by glycine. Am. J. Physiol. 277, L952-L959 (1999).

34. Pless, S. A., Dibas, M. I., Lester, H. A. \& Lynch, J. W. Conformational variability of the glycine receptor $\mathrm{M} 2$ domain in response to activation by different agonists. J. Biol. Chem. 282, 36057-36067 (2007).

35. Alexander, S. P. et al. The concise guide to pharmacology 2017/18: ligandgated ion channels. Br. J. Pharmacol. 174(Suppl 1), S130-S159 (2017).

36. Wheeler, M. et al. Glycine-gated chloride channels in neutrophils attenuate calcium influx and superoxide production. FASEB J. 14, 476-484 (2000).

37. Ikejima, K., Qu, W., Stachlewitz, R. F. \& Thurman, R. G. Kupffer cells contain a glycine-gated chloride channel. Am. J. Physiol. 272, G1581-G1586 (1997).

38. Betz, H. \& Becker, C. M. The mammalian glycine receptor: biology and structure of a neuronal chloride channel protein. Neurochem. Int. 13, 137-146 (1988).

39. Mulhardt, C. et al. The spastic mouse: aberrant splicing of glycine receptor beta subunit mRNA caused by intronic insertion of L1 element. Neuron 13 1003-1015 (1994)

40. Bormann, J., Rundstrom, N., Betz, H. \& Langosch, D. Residues within transmembrane segment $\mathrm{M} 2$ determine chloride conductance of glycine receptor homo- and hetero-oligomers. EMBO J. 12, 3729-3737 (1993).

41. Maksay, G., Laube, B. \& Betz, H. Subunit-specific modulation of glycine receptors by neurosteroids. Neuropharmacology 41, 369-376 (2001).

42. Majewska, M. D., Mienville, J. M. \& Vicini, S. Neurosteroid pregnenolone sulfate antagonizes electrophysiological responses to GABA in neurons. Neurosci. Lett. 90, 279-284 (1988).

43. Hawthorne, R., Cromer, B. A., Ng, H. L., Parker, M. W. \& Lynch, J. W. Molecular determinants of ginkgolide binding in the glycine receptor pore. J. Neurochem. 98, 395-407 (2006).

44. Huang, S. H. et al. Ginkgolides, diterpene trilactones of Ginkgo biloba, as antagonists at recombinant alpha1beta2gamma2L GABAA receptors. Eur. J. Pharmacol. 494, 131-138 (2004).

45. Johnston, G. A. Muscimol as an ionotropic GABA receptor agonist. Neurochem. Res. 39, 1942-1947 (2014).

46. Weinberg, J. M., Venkatachalam, M. A., Garzo-Quintero, R., Roeser, N. F. \& Davis, J. A. Structural requirements for protection by small amino acids against hypoxic injury in kidney proximal tubules. FASEB J. 4, 3347-3354 (1990).
47. Dong, Z., Venkatachalam, M. A., Weinberg, J. M., Saikumar, P. \& Patel, Y. Protection of ATP-depleted cells by impermeant strychnine derivatives: implications for glycine cytoprotection. Am. J. Pathol. 158, 1021-1028 (2001).

48. Aleo, M. D. \& Schnellmann, R. G. The neurotoxicants strychnine and bicuculline protect renal proximal tubules from mitochondrial inhibitor-induced cell death. Life Sci. 51, 1783-1787 (1992).

49. Venkatachalam, M. A., Weinberg, J. M., Patel, Y., Saikumar, P. \& Dong, Z. Cytoprotection of kidney epithelial cells by compounds that target amino acid gated chloride channels. Kidney Int. 49, 449-460 (1996).

50. Waters, S. L., Miller, G. W., Aleo, M. D. \& Schnellmann, R. G. Neurosteroid inhibition of cell death. Am. J. Physiol. 273, F869-F876 (1997).

51. Marvizon, J. C., Lewin, A. H. \& Skolnick, P. 1-aminocyclopropane carboxylic acid: a potent and selective ligand for the glycine modulatory site of the N-methylD-aspartate receptor complex. J. Neurochem. 52, 992-994 (1989).

52. OToole, K. K. \& Jenkins, A. The apparent voltage dependence of GABAA receptor activation and modulation is inversely related to channel open probability. Mol. Pharmacol. 81, 189-197 (2012).

53. Sauguet, L. et al. Structural basis for potentiation by alcohols and anaesthetics in a ligand-gated ion channel. Nat. Commun. 4, 1697 (2013).

54. Yevenes, G. E. \& Zeilhofer, H. U. Allosteric modulation of glycine receptors. Br. J. Pharmacol. 164, 224-236 (2011).

55. Olsen, R. W. GABAA receptor: positive and negative allosteric modulators. Neuropharmacology 136, 10-22 (2018).

56. Evavold, C. L. et al. The pore-forming protein gasdermin D regulates interleukin-1 secretion from living macrophages. Immunity 48, 35-44 (2018).

57. Schneider, K. S. et al. The inflammasome drives GSDMD-independent secondary pyroptosis and IL-1 release in the absence of caspase- 1 protease activity. Cell Rep. 21, 3846-3859 (2017).

58. Ahrens, S. et al. F-actin is an evolutionarily conserved damage-associated molecular pattern recognized by DNGR-1, a receptor for dead cells. Immunity 36, 635-645 (2012).

59. Zhang, J. G. et al. The dendritic cell receptor Clec9A binds damaged cells via exposed actin filaments. Immunity 36, 646-657 (2012).

60. Maeda, A. \& Fadeel, B. Mitochondria released by cells undergoing TNFalpha-induced necroptosis act as danger signals. Cell Death Dis. 5, e1312 (2014).

61. Franklin, B. S. et al. The adaptor ASC has extracellular and 'prionoid' activities that propagate inflammation. Nat. Immunol. 15, 727-737 (2014).

62. Baroja-Mazo, A. et al. The NLRP3 inflammasome is released as a particulate danger signal that amplifies the inflammatory response. Nat. Immunol. 15 738-748 (2014).

63. Jorgensen, I., Zhang, Y., Krantz, B. A. \& Miao, E. A. Pyroptosis triggers poreinduced intracellular traps (PITs) that capture bacteria and lead to their clearance by efferocytosis. J. Exp. Med. 213, 2113-2128 (2016).

64. Kang, R. et al. Lipid peroxidation drives gasdermin D-mediated pyroptosis in lethal polymicrobial sepsis. Cell Host Microbe 24,97-108 (2018). 